Article

\title{
Environmental Health-Related Policies and Practices of Oklahoma Licensed Early Care and Education Programs: Implications for Childhood Asthma
}

\author{
Cassandra D. Querdibitty ${ }^{1}$, Bethany Williams ${ }^{2,3}{ }^{\circledR}$, Marianna S. Wetherill ${ }^{1} \mathbb{D}$, Susan B. Sisson ${ }^{3}$, \\ Janis Campbell $\left.{ }^{4}{ }^{(}\right)$, Mary Gowin ${ }^{5}$, Lancer Stephens ${ }^{1}$ and Alicia L. Salvatore ${ }^{1,6, *}$
}

check for updates

Citation: Querdibitty, C.D.; Williams, B.; Wetherill, M.S.; Sisson, S.B.; Campbell, J.; Gowin, M.; Stephens, L.; Salvatore, A.L. Environmental Health-Related Policies and Practices of Oklahoma Licensed Early Care and Education Programs: Implications for Childhood Asthma. Int. J. Environ. Res. Public Health 2021, 18, 8491. https://doi.org/ $10.3390 /$ ijerph18168491

Academic Editor: Paul B. Tchounwou

Received: 14 June 2021

Accepted: 6 August 2021

Published: 11 August 2021

Publisher's Note: MDPI stays neutral with regard to jurisdictional claims in published maps and institutional affiliations.

Copyright: (c) 2021 by the authors. Licensee MDPI, Basel, Switzerland. This article is an open access article distributed under the terms and conditions of the Creative Commons Attribution (CC BY) license (https:/ / creativecommons.org/licenses/by/ $4.0 /)$.
1 Department of Health Promotion Sciences, Hudson College of Public Health, University of Oklahoma Health Sciences Center, 801 N.E. 13th Street, Oklahoma City, OK 73104, USA; cassandra-querdibitty@ouhsc.edu (C.D.Q.); Marianna-Wetherill@ouhsc.edu (M.S.W.); Lancer-Stephens@ouhsc.edu (L.S.)

2 Department of Nutrition and Exercise Physiology, Elson S. Floyd College of Medicine, Washington State University Health Sciences Spokane, 412 E. Spokane Falls Blvd., Spokane, WA 99202, USA; bethany.williams1@wsu.edu

3 Department of Nutritional Sciences, College of Allied Health, University of Oklahoma Health Sciences Center, 1200 N. Stonewall Ave., Oklahoma City, OK 73114, USA; Susan-Sisson@ouhsc.edu

4 Department of Biostatistics and Epidemiology, Hudson College of Public Health, University of Oklahoma Health Sciences Center, 801 N.E. 13th Street, Oklahoma City, OK 73104, USA; Janis-Campbell@ouhsc.edu

5 Department of Family \& Preventive Medicine, College of Medicine, University of Oklahoma, 900 N.E. 10th Street, Oklahoma City, OK 73104, USA; Mary-Gowin@ouhsc.edu

6 Institute for Research on Equity and Community Health (iREACH), ChristianaCare, Avenue North, 4000 Nexus Drive, CEI-300, Wilmington, DE 19803, USA

* Correspondence: alicia.salvatore@christianacare.org

Abstract: Little is known about the environmental health-related policies and practices of early care and education (ECE) programs that contribute to childhood asthma, particularly in Oklahoma where child asthma rates $(9.8 \%)$ and rates of uncontrolled asthma among children with asthma $(60.0 \%)$ surpass national rates ( $8.1 \%$ and $50.3 \%$, respectively). We conducted a cross-sectional survey with directors of Oklahoma-licensed ECE programs to assess policies and practices related to asthma control and to evaluate potential differences between Centers and Family Childcare Homes (FCCHs). Surveyed ECEs $(n=476)$ included Centers $(56.7 \%)$, FCCHs $(40.6 \%)$, and other program types $(2.7 \%)$. Almost half (47.2\%) of directors reported never receiving any asthma training. More Center directors were asthma-trained than FCCH directors $(61.0 \%$ versus $42.0 \%, p<0.0001)$. Most ECEs used asthma triggers, including bleach $(88.5 \%)$ and air fresheners (73.6\%). Centers were more likely to use bleach daily than were FCCHs $(75.6 \%$ versus $66.8 \%, p=0.04)$. FCCHs used air fresheners more than did Centers $(79.0 \%$ versus $61.0 \%, p<0.0001)$. The majority of ECEs $(74.8 \%)$ used pesticides indoors. Centers applied indoor pesticides more frequently (i.e., monthly or more often) than did FCCHs $(86.0 \%$ versus $58.0 \%, p<0.0001)$. Policy, educational, and technical assistance interventions are needed to reduce asthma triggers and improve asthma control in Oklahoma ECEs.

Keywords: Oklahoma; childcare; children's environmental health; allergens; pesticides; chemicals; asthma; policy

\section{Introduction}

Early care and education (ECE) programs, also known as childcare programs, are critical environments that shape children's health. Approximately 13 million (61\%) children under the age of five in the United States (US) receive regular care in ECE programs [1], for as much as $50 \mathrm{~h}$ a week [2,3]. Young children attending ECE programs can be chronically exposed to environmental toxicants from commonly used products, such as cleaners, air 
fresheners, and pesticides, at ECEs [4-9]. These products may emit volatile organic compounds (VOCs) into the indoor air that can then interact with other chemicals to produce secondary pollutants known as particulate matter (PM) $[10,11]$. PM is a complex mixture of particles and liquid droplets including nitrates, sulfates, elemental and organic carbon, organic compounds (e.g., polycyclic aromatic hydrocarbons), biological compounds (e.g., endotoxin and cell fragments), and metals (e.g., iron, copper, nickel, zinc, and vanadium) in suspended air of various sizes of concern including "inhalable coarse particles" with a diameter of 2.5 to $10 \mu \mathrm{m}\left(\mathrm{PM}_{10}\right)$, "fine particles" smaller than $2.5 \mu \mathrm{m}$ in diameter $\left(\mathrm{PM}_{2.5}\right)$, and "ultrafine particles" with a diameter less than $0.1 \mu \mathrm{m}\left(\mathrm{PM}_{0.1}\right)[12,13]$. Children's small body size, unique behaviors and activity levels, physiology, and rapid development make them particularly vulnerable to these exposures and their potential health effects $[14,15]$. Children have higher respiratory rates, breathe a larger volume of air per unit body weight [16], and have increased physical exertion that results in greater inhalation of potentially harmful chemicals [17]. Childhood exposures to VOCs, PM, and nitrogen dioxide have been associated with adverse respiratory outcomes including decreased lung function, inflammation, and airway obstruction; increased allergen sensitization; and the exacerbation of asthma symptoms $[15,18-23]$.

Environmental toxicants in indoor environments are dependent on numerous factors including the age [24] and structural quality of the building or facility [25-28], maintenance, and cleaning practices $[10,29,30]$. To illustrate, a building that is poorly maintained may be more susceptible to mold and pests potentially leading to the frequent use of pesticides [8], which were associated with higher indoor levels of VOCs [11,31-33]. While limited, studies have documented the presence of VOCs and PM (i.e., $\mathrm{PM}_{2.5}$ and $\mathrm{PM}_{10}$ ) in ECE settings [4,8,34]. A study in northern California [4] found several environmental toxicants in childcare facilities such as chloroform, benzene, and ethylbenzene, at estimated concentrations exceeding government health-based exposure levels. Those chemicals that reached exposure levels of concern were known carcinogens and endocrine disruptors. Additionally, these chemicals may exacerbate asthma and other respiratory illnesses and may disrupt neurocognitive functioning among children. Notably, a study of childcare centers in inner-city Washington D.C. [8], found a maximum concentration of commonly detected VOCs were over 14 times higher than those reported in the California study. Exposures to pest allergens such as cockroaches, mice, dust mites, and rodents have been associated with sensitization and are common triggers of childhood asthma symptoms [35-38]. A majority of ECEs in two North Carolina counties detected cockroach and mouse allergen, $52 \%$ and $83 \%$ respectively [39]. Similarly, a study in Arkansas, found $100 \%$ of Head Starts had detectable mouse allergens [40]. A combination of chemical and non-chemical pest control methods, also known as integrated pest management (IPM), aims to eliminate the source using pesticides and maintain low levels of pest allergens using less/non-toxic alternatives [41-44]. However, childcare centers revealed high management and staff turnover, hectic work environment, and inadequate coordination with cleaning and pest management contractors made it difficult to implement IPM strategies [45]. Organophosphorus and pyrethroids pesticides, known neurotoxins, are commonly detected indoors and [9] routinely applied in classrooms and on playgrounds [46]. Pesticide residues can remain on surfaces children frequently encounter (e.g., floors, carpets, furniture, toys) and accumulate in dust that can easily be inhaled or ingested by children [47].

ECE programs' environmental health-related policies and practices may influence children's exposures to environmental toxicants and subsequent risk of asthma attacks and adverse health outcomes. Understanding the types of policies and practices carried out by ECE programs is therefore paramount to creating healthier ECE environments. Yet, little is known about the environmental health-related policies and practices of ECE programs. Most studies to date with notable exceptions [8,48,49] were conducted in California [50-53], the state with the most protective policies, and most research was carried out in childcare centers (i.e., federally-supported Head Start programs and community-based childcare). Little research has been conducted in other geographical regions of the US or in family 
childcare homes (FCCHs) (i.e., home-based childcare). Centers and FCCHs' environmental health-related policies and practices, including those related to childhood asthma control, may differ largely due to their setting and participation in accrediting bodies. Accrediting bodies, such as the National Association for the Education of Young Children (NAEYC), have standards that are generally more rigorous than those of state licensing requirements. The NAEYC has specific accreditation assessment items related to environmental health, including (1) air fresheners; (2) fragrance-free and least-toxic cleaning products; and (3) non-toxic pest management techniques (i.e., IPM) [54]. IPM is a safer approach to controlling pests because its strategies focus on preventing infestations by monitoring pests and limiting the use of harmful pesticides [55]. Regardless of the type of ECE program, all children should receive care in a healthy childcare environment that reduces asthma triggers and improves asthma control.

The prevalence of childhood asthma in Oklahoma $(9.8 \%)$ is higher than the national childhood asthma prevalence (8.1\%) [56]. The rate of uncontrolled asthma among children with asthma in Oklahoma (60.0\%) also exceeds the national rate (50.3\%) [57]. In Oklahoma and in many other states, young children primarily receive childcare in one of two settings: Centers and FCCHs. Centers are usually located in larger facilities (i.e., commercial buildings), have multiple classes and larger sizes, and have multiple staff members [58]. FCCHs care for 12 or fewer children, are located in family homes or residences, and have a single provider, sometimes with an assistant [59].

The purpose of the present study was to characterize environmental health-related policies and practices of licensed ECE programs in Oklahoma caring for preschool-aged children (i.e., three to five years old). We examined policies and practices for Oklahoma ECE programs overall and also assessed differences between the two primary ECE program types: (1) Centers and (2) FCCHs. Our study focused on policies and practices in three key areas: (1) asthma training, prevention, and control; (2) cleaners and air fresheners; and (3) pesticides and pest control methods.

\section{Materials and Methods}

\subsection{Study Design}

The Communities and Classroom Health Survey, a cross-sectional survey of Oklahoma ECE directors, was conducted from November 2019 through February 2020. Eligible ECE programs were (1) licensed in Oklahoma and (2) cared for preschool-aged children. Our total sampling frame of existing Oklahoma-licensed ECE programs $(n=3121)$ included Head Starts $(n=343)$, community-based childcare (i.e., CBCC; $n=1130$ ), and FCCHs $(n=1648)$. Program directors were targeted a priori as the primary survey participants; however, if preferred by the director, another staff member who was knowledgeable about the program's policies or practices could participate. A total of 159 programs were excluded because (1) program approval was not obtained, (2) programs were Tribally-owned ECEs operated by a sovereign tribal nation with an Institutional Review Board, (3) programs did not care for preschool-aged children, or (4) programs were deemed to be duplicates. Of the 2962 programs that were eligible and invited to participate in the study, 191 were Head Starts, 1126 were CBCCs, and 1645 were FCCHs. Detailed methods on the survey and recruitment were previously published [60]. For the purpose of the current study, participating programs were categorized into one of two primary ECE types: (1) Centerbased childcare or "Centers" (i.e., Head Starts and CBCCs) or (2) FCCHs. The OUHSC Institutional Review Board deemed this program-level study exempt. This was due to data being program-level, not individual-level; thus, this study was not considered as human-subjects research.

\subsection{Data Collection}

Contact information was obtained from a current census of Oklahoma Head Start programs (provided by the Oklahoma Head Start Collaboration Office) and an up-to-date registry of CBCCs and FCCHs (provided by the Oklahoma Department of Health). In 
November 2019, eligible ECE programs were mailed a survey packet with (1) a cover letter with detailed consent instructions; (2) a survey booklet with instructions on how to complete the survey; (3) an optional link to complete the survey online using Research Electronic Data Capture (REDCap) [61,62]; and (4) a postage-paid business reply envelope for directors who preferred to complete the survey on paper. A reminder postcard was mailed to non-respondents in December 2019. A second and final round of survey packets was mailed out to non-respondents in January 2020. Reminder phone calls were made to non-respondents using publicly available telephone numbers in January and February 2020. Participants could enter a raffle for a $\$ 20$ Amazon gift card for completing the survey. Identifiable information provided to participate in the raffle was deidentified and was not linked with survey responses.

\subsection{Measures}

\subsubsection{Program Characteristics}

Directors reported ECE program-level characteristics, including program type (i.e., Centers versus FCCHs). For analyses, ECE program type was collapsed into three categories: (1) Center (i.e., "center-based childcare" and "Head Start"), (2) FCCH (i.e., "family childcare home"), and (3) Other (i.e., "public pre-k program"). Public pre-k programs, which are typically a part of public-school systems and are privy to different policies and resources, were categorized as Other and were only included in analyses for the total sample. Programs were also classified by NAEYC accreditation status (yes/no).

\subsubsection{Asthma-Related Training, Policies, and Practices}

Items were adapted from the Preparing Asthma in Child Care (PACC) Instrument [63], which was designed to measure the preparedness of ECE programs to prevent and manage asthma exacerbations via asthma training for staff and asthma-related policies and guidelines. Types of asthma training assessed included (1) asthma basics (i.e., causes of asthma, signs of asthma flare-ups), (2) reducing asthma allergens and irritants, (3) asthma medication use and types, (4) asthma management plans, and (5) proper administration of asthma medications. Policies and guidelines assessed included (1) managing asthma medications and (2) reducing asthma allergens and irritants. Additional questions used to determine the presence of known asthma allergens included whether the program had: (1) any pets (e.g., cats, dogs, gerbils, or birds), (2) wall-to-wall carpet, and (3) staff that smoked or vaped on facility property.

\subsubsection{Bleach and Air Fresheners}

Items were also adapted from the Environmental Exposures in Child Care Facilities Study [4] to measure the usage of (1) bleach, (2) less toxic cleaners, and (3) air fresheners.

\subsubsection{Pesticide Use and Pest Control}

Items were adapted from the Pest Management and Pesticide Use in California Child Care Centers questionnaire to assess pest exposure, pest management, and pesticide use [64]. Indoor and outdoor pest problems in the past 12-months were measured using two questions with multi-select response options. Two questions were used to measure the frequency of pesticide use inside and outside the ECE facility in the past 12 months, including the use of pesticide sprays, scatters, and bombs. Two separate questions assessed who applied pesticides inside and outside of the ECE including multi-select response options. The presence of an ECE policy regarding pesticide use (when and how) was measured with a single question. Staff and parental notification prior to pesticide application inside or outside of the ECE were assessed by two questions. See Supplementary Material Figure S1 for a copy of all survey questions. 


\subsection{Data Analyses}

Paper survey responses were entered into a REDCap database and combined with online survey responses. SAS version 9.4 (SAS Institute Inc., Cary, NC, USA) was used for all analyses. Descriptive statistics were conducted on all measures, including mean and standard deviations (SDs) for continuous variables and frequencies and percentages for categorical or nominal variables. Analyses were conducted with the total sample, then stratified by program type (i.e., Centers and FCCHs). The differences between Centers and FCCHs were evaluated with Chi-square analyses, Fisher's exact tests for variables with more than $20 \%$ of cells with expected frequencies $<5$, and $t$-tests for continuous variables. Statistical significance was set at $p<0.05$. The initial significance level of 0.05 was adjusted to 0.025 for multiple hypothesis testing using the Bonferroni method [65].

Stratification by NAEYC accreditation, as a proxy to star rating, precluded analyses due to the small sample size $(n=58)$. A sensitivity analysis, however, was conducted to explore possible differences by NAEYC accreditation.

\section{Results}

\subsection{Program Characteristics}

The overall response rate was $16.0 \%$ and included 476 surveys (Figure 1), with $33.5 \%$ Head Starts, $18.3 \%$ CBCCs, and $11.7 \%$ FCCHs responding.
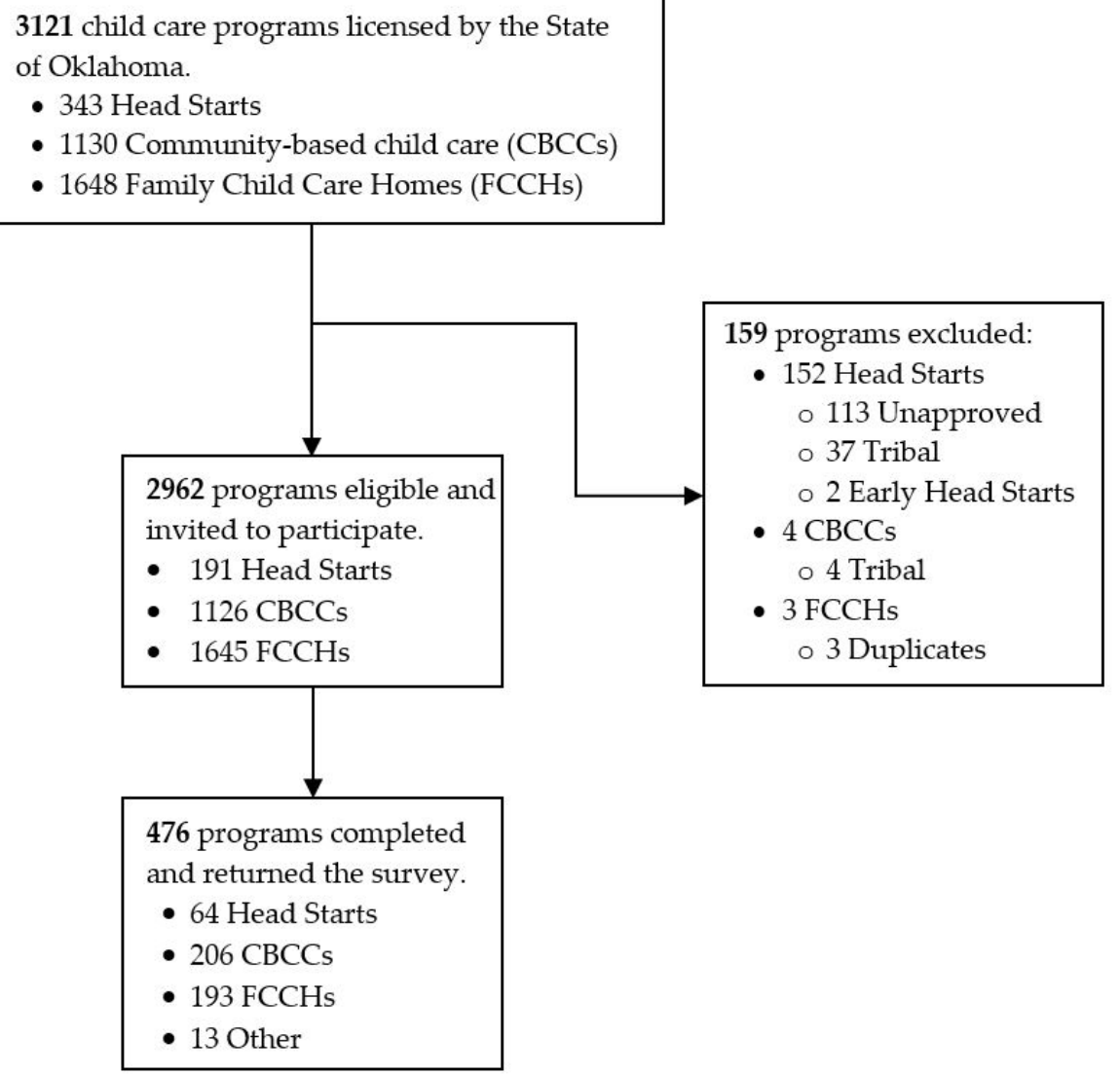

Figure 1. Program participation in the Communities and Classroom Health Survey $(n=476)$, Oklahoma 2019-2020.

Of the 476 participating ECE programs, a little over half (56.7\%) were Centers (Table 1). Most programs (92.7\%) were in operation for a full day and were fully enrolled (66.2\%). Few programs were NAEYC accredited (12.2\%). The average number of preschool classrooms was about two $(S D=2.4)$. Centers typically had about three preschool classrooms $(S D=2.8$; Range: 1-34) and FCCHs had about one ( $S D=1.4$; Range: $1-2)$. While Centers cared for 
an average of about 34 preschool-aged children $(S D=46.5$; Range: $2-574)$, FCCHs had an average of fewer than four $(M=3.7 ; S D=2.4$; Range: $0-20)$.

Table 1. Early care and education program characteristics $(n=476)$, Oklahoma 2019-2020.

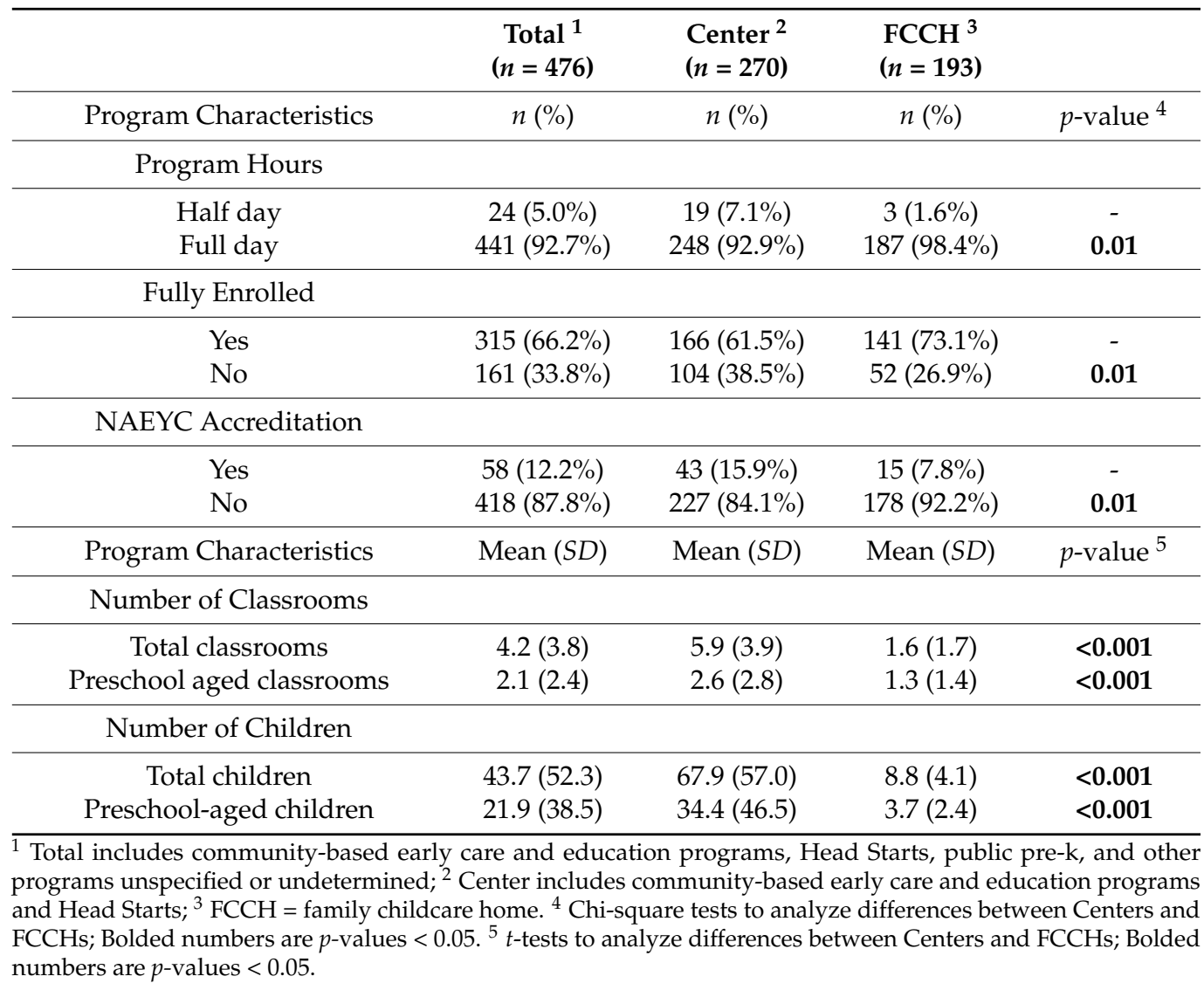

\subsection{Asthma-Related Training, Policies and Practices}

About half (52.7\%) of directors reported ever receiving any asthma training (Table 2). Center directors $(61.0 \%)$ were more likely to report any asthma training than were FCCH directors $(42.0 \%)(p<0.0001)$, and were more likely to report training across all four training areas: (1) asthma basics $(49.0 \%$ versus $36.0 \%, p=0.01)$, (2) asthma medication use and types $(37.0 \%$ versus $19.0 \%, p<0.0001)$, (3) asthma management plans $(17.0 \%$ versus $19.0 \%, p=0.02)$, and (4) proper administration of asthma medications $(42.0 \%$ versus $26.0 \%$, $p<0.001)$. While most $(82.4 \%)$ programs had policies/guidelines for managing asthma medications, Centers were more likely than FCCHs to have such policies/guidelines $(88.0 \%$ versus $68.0 \%, p<0.0001)$. Over one-in-four of programs $(27.3 \%)$ overall had pets at their facilities. Over half of FCCHs $(57 \%)$ had pets, while few $(7 \%)$ Centers did $(p<0.0001)$. About one in ten (10.1\%) programs reported that staff smoked or vaped on facility property. Centers, however, were more likely to report that staff smoked or vaped on facility property, albeit outside only, than were FCCHs $(14.0 \%$ versus $5.0 \%, p<0.001)$. 
Table 2. Asthma-related training, policies, and practices reported for all early care and education programs, Centers, and family childcare homes (FCCHs), Oklahoma 2019-2020.

\begin{tabular}{|c|c|c|c|c|}
\hline Survey Items & $\begin{array}{c}\text { Total } \\
(n=476)\end{array}$ & $\begin{array}{l}\text { Center } \\
(n=270)\end{array}$ & $\begin{array}{l}\text { FCCH } \\
(n=193)\end{array}$ & \\
\hline & $n(\%)$ & $n(\%)$ & $n(\%)$ & $p$-Value ${ }^{1}$ \\
\hline \multicolumn{5}{|l|}{ Ever received any type of asthma training } \\
\hline Yes & $251(52.7 \%)$ & $166(61.0 \%)$ & $81(42.0 \%)$ & - \\
\hline No & $225(47.3 \%)$ & $104(39.0 \%)$ & $112(58.0 \%)$ & $<0.0001$ \\
\hline \multicolumn{5}{|l|}{ Type of asthma training received } \\
\hline Asthma basics & - & - & - & - \\
\hline Yes & $204(42.9 \%)$ & $131(49.0 \%)$ & $70(36.0 \%)$ & - \\
\hline No & $272(57.1 \%)$ & $139(51.0 \%)$ & $123(64.0 \%)$ & 0.01 \\
\hline Reducing asthma allergens and irritants & - & - & - & - \\
\hline Yes & $88(18.5 \%)$ & $58(21.0 \%)$ & $28(15.0 \%)$ & - \\
\hline No & $388(81.5 \%)$ & $212(79.0 \%)$ & $165(85.0 \%)$ & 0.06 \\
\hline Asthma medications use and types & - & - & - & - \\
\hline Yes & $139(29.2 \%)$ & $99(37.0 \%)$ & $37(19.0 \%)$ & - \\
\hline No & $337(70.8 \%)$ & $171(63.0 \%)$ & $156(81.0 \%)$ & $<0.0001$ \\
\hline Asthma management plans & - & - & - & - \\
\hline Yes & $65(13.7 \%)$ & $46(17.0 \%)$ & $18(9.0 \%)$ & - \\
\hline No & $411(86.3 \%)$ & $224(83.0 \%)$ & $175(91.0 \%)$ & 0.02 \\
\hline Proper administration of asthma medications & - & - & - & - \\
\hline Yes & $170(35.75 \%)$ & $116(43.0 \%)$ & $50(26.0 \%)$ & - \\
\hline No & $306(64.3 \%)$ & $154(57.0 \%)$ & $143(74.0 \%)$ & $<0.0001$ \\
\hline \multicolumn{5}{|l|}{ Policies/guidelines about managing asthma medications } \\
\hline Yes & $378(82.4 \%)$ & $237(88.0 \%)$ & $132(68.0 \%)$ & - \\
\hline No & $81(17.7 \%)$ & $33(12.0 \%)$ & $61(32.0 \%)$ & $<0.0001$ \\
\hline \multicolumn{5}{|l|}{$\begin{array}{c}\text { Policies/guidelines about reducing asthma allergens and } \\
\text { irritants }\end{array}$} \\
\hline Yes & $175(36.8 \%)$ & $109(40.0 \%)$ & $63(33.0 \%)$ & - \\
\hline No & $301(63.2 \%)$ & $161(60.0 \%)$ & $130(67.0 \%)$ & 0.09 \\
\hline \multicolumn{5}{|l|}{ Pets present at facility } \\
\hline Yes & $130(27.3 \%)$ & $19(7.0 \%)$ & $110(57.0 \%)$ & - \\
\hline No & $346(72.7 \%)$ & $251(93.0 \%)$ & $83(43.0 \%)$ & $<0.0001$ \\
\hline \multicolumn{5}{|l|}{ Facility has wall-to-wall carpet } \\
\hline Yes & $120(25.2 \%)$ & $60(22.0 \%)$ & $58(30.0 \%)$ & - \\
\hline No & $356(74.8 \%)$ & $210(78.0 \%)$ & $135(70.0 \%)$ & 0.06 \\
\hline \multicolumn{5}{|l|}{ Staff smoke or vape on facility property } \\
\hline Yes, but outside only & $48(10.1 \%)$ & $39(14.0 \%)$ & $9.0(5.0 \%)$ & $<0.0001$ \\
\hline Yes, both inside and outside & $0(0.0 \%)$ & $0(0.0 \%)$ & $0(0.0 \%)$ & - \\
\hline No & $428(89.9 \%)$ & $231(86.0 \%)$ & $184(95.0 \%)$ & - \\
\hline
\end{tabular}

${ }^{1}$ Chi-square tests to analyze differences between Centers and FCCHs; Bolded numbers are $p$-values $<0.05$.

\subsection{Bleach and Air Fresheners}

Most (88.5\%) programs reported using bleach at their facilities (Table 3), with Centers being more likely than FCCHs to use bleach daily or a few times a day $(76.0 \%$ versus $67.0 \%$, $p=0.04)$. A majority (62.0\%) of programs reported that they used "low toxicity" or "less toxic" cleaners, with Centers less likely to use these cleaners than FCCHs $(58.0 \%$ versus $68.0 \%, p=0.03)$. A large proportion (73.6\%) of programs reported using air fresheners. Centers were less likely to use air fresheners compared to FCCHs $(61.0 \%$ versus $79.0 \%$, $p<0.0001)$. Specifically, Centers were less likely to use scented candles $(2.0 \%$ versus $19.0 \%$, 
$p<0.0001)$, continuous-release air fresheners $(19.0 \%$ versus $31.0 \%, p=0.001)$, and essential oil diffusers $(11.0 \%$ versus $20.0 \%, p=0.01)$ than were FCCHs.

Table 3. Reported use of bleach, air fresheners, and cleaners for all early care and education programs, centers, and family childcare homes (FCCHs), Oklahoma 2019-2020.

\begin{tabular}{|c|c|c|c|c|}
\hline Survey Items & $\begin{array}{c}\text { Total } \\
(n=476)\end{array}$ & $\begin{array}{c}\text { Center } \\
(n=270)\end{array}$ & $\begin{array}{c}\text { FCCH } \\
(n=193)\end{array}$ & \\
\hline & $n(\%)$ & $n(\%)$ & $n(\%)$ & $p$-Value ${ }^{1}$ \\
\hline \multicolumn{5}{|l|}{ Use bleach at facility } \\
\hline Yes & $421(88.5 \%)$ & $233(86.0 \%)$ & $178(92.0 \%)$ & - \\
\hline No & $55(11.6 \%)$ & $37(14.0 \%)$ & $15(8.0 \%)$ & 0.05 \\
\hline \multicolumn{5}{|l|}{ Frequency of bleach use at facility } \\
\hline Every few months or less often & - & - & - & - \\
\hline Yes & $15(3.2 \%)$ & $6(2.2 \%)$ & $7(3.6 \%)$ & - \\
\hline No & $461(96.9 \%)$ & $264(97.8 \%)$ & $186(96.4 \%)$ & 0.37 \\
\hline Monthly or a few times a month & - & - & - & - \\
\hline Yes & $17(3.6 \%)$ & $8(3.0 \%)$ & $9(4.7 \%)$ & - \\
\hline No & $459(96.4 \%)$ & $262(97.0 \%)$ & $184(95.3 \%)$ & 0.34 \\
\hline Weekly or a few times a week & - & - & - & - \\
\hline Yes & $49(10.3 \%)$ & $15(5.6 \%)$ & $33(17.1 \%)$ & - \\
\hline No & $427(89.7 \%)$ & $255(94.4 \%)$ & $160(82.9 \%)$ & $<0.0001$ \\
\hline Daily or a few times a day & - & - & - & - \\
\hline Yes & $340(71.4 \%)$ & $204(75.6 \%)$ & $129(66.8 \%)$ & - \\
\hline No & $136(28.6 \%)$ & $66(24.4 \%)$ & $64(33.2 \%)$ & 0.04 \\
\hline \multicolumn{5}{|l|}{ Use "low toxicity" or "less toxic" cleaners at facility } \\
\hline Yes & $295(62.0 \%)$ & $156(58.0 \%)$ & $131(68.0 \%)$ & - \\
\hline No & $181(38.0 \%)$ & $114(42.0 \%)$ & $62(32.0 \%)$ & 0.03 \\
\hline \multicolumn{5}{|l|}{ Use air fresheners at facility } \\
\hline Yes & $323(73.6 \%)$ & $164(61.0 \%)$ & $153(79.0 \%)$ & - \\
\hline No & $116(26.4 \%)$ & $106(39.0 \%)$ & $40(21.0 \%)$ & $<0.0001$ \\
\hline \multicolumn{5}{|l|}{ Types of air fresheners used } \\
\hline Scented candles & $42(8.8 \%)$ & $6(2.0 \%)$ & $36(19.0 \%)$ & $<0.0001$ \\
\hline Spray air fresheners & $190(39.9 \%)$ & $106(39.0 \%)$ & $81(42.0 \%)$ & 0.56 \\
\hline Continuous release (such as a plug-in) & $112(23.5 \%)$ & $52(19.0 \%)$ & $59(31.0 \%)$ & $<0.0001$ \\
\hline Incense & $6(1.3 \%)$ & $2(1.0 \%)$ & $4(2.0 \%)$ & 0.24 \\
\hline Essential oils (reed diffuser or other type of diffuser) & $73(15.3 \%)$ & $34(13.0 \%)$ & $36(19.0 \%)$ & 0.07 \\
\hline Essential oils electric or battery diffuser & $71(14.9 \%)$ & $31(11.0 \%)$ & $39(20.0 \%)$ & 0.01 \\
\hline Potpourri & $1(0.2 \%)$ & $0(0.0 \%)$ & $1(1.0 \%)$ & 0.42 \\
\hline Gel canister & $10(2.1 \%)$ & $6(2.0 \%)$ & $3(2.0 \%)$ & 0.74 \\
\hline Scented wax melts & $25(5.3 \%)$ & $10(4.0 \%)$ & $15(8.0 \%)$ & 0.06 \\
\hline Other types & $13(2.7 \%)$ & $5(2.0 \%)$ & $8(4.0 \%)$ & 0.14 \\
\hline
\end{tabular}

${ }^{1}$ Chi-square tests to analyze differences between Centers and FCCHs; Bolded numbers $p$-values $<0.05$; Fisher's exact tests to analyze differences between Centers and FCCHs for variable with a cell size $<5$; Initial significance level of 0.05 was adjusted to 0.025 for multiple hypothesis testing using the Bonferroni method.

\subsection{Pesticide Use and Pest Control}

About a quarter (23.5\%) of ECEs reported that pesticides were sprayed, scattered, or "bombed" inside their facility weekly or monthly (Table 4), with these applications more common in Centers than FCCHs $(35.0 \%$ versus $6.0 \%, p<0.001)$. About half $(49.0 \%)$ of programs used a pest control company to apply pesticides inside their facilities. Centers used pest control companies for indoor pesticide applications more often than did FCCHs $(64.0 \%$ versus $27.0 \%, p<0.001)$. About one-in-six $(15.6 \%)$ programs reported pesticide use outside their facility weekly or monthly. Centers reported more frequent outdoor pesticide applications than did FCCHs $(20.0 \%$ versus $8.0 \%, p<0.001)$. Less than half $(43.3 \%)$ of 
programs used a pest control company for outdoor pesticide applications at their facilities, with Centers reporting doing so more often than FCCHs $(51.0 \%$ versus $32.0 \%, p<0.0001$ ). A small proportion $(21.8 \%)$ of ECE programs had a written policy about pesticide use. Centers were more likely than FCCHs to have a written pesticide use policy ( $31.0 \%$ versus $8.0 \%, p<0.0001)$. Over one-in-three $(36.7 \%)$ programs reported notifying parents before applying pesticides. Centers (29.0\%), however, were less likely than FCCHs $(42.0 \%)$ to notify parents before pesticide applications inside or outside their facility $(p=0.01)$.

Table 4. Reported pest problems, pest control methods, applicators, frequency, and related topics for all early care and education programs, centers, and family childcare homes (FCCHs), Oklahoma 2019-2020.

\begin{tabular}{|c|c|c|c|c|}
\hline Survey Items & $\begin{array}{c}\text { Total } \\
(n=476)\end{array}$ & $\begin{array}{c}\text { Center } \\
(n=270)\end{array}$ & $\begin{array}{c}\text { FCCH } \\
(n=193)\end{array}$ & \\
\hline & $n(\%)$ & $n(\%)$ & $n(\%)$ & $p$-Value ${ }^{1}$ \\
\hline \multicolumn{5}{|l|}{ Have indoor pest problem(s) } \\
\hline Yes & $225(47.3 \%)$ & $142(53.0 \%)$ & $78(40.0 \%)$ & - \\
\hline No & $251(52.7 \%)$ & $128(47.0 \%)$ & $115(60.0 \%)$ & 0.01 \\
\hline \multicolumn{5}{|l|}{ Have outdoor pest problem(s) } \\
\hline Yes & $271(56.9 \%)$ & $153(57.0 \%)$ & $114(59.0 \%)$ & - \\
\hline No & $205(43.1 \%)$ & $117(43.0 \%)$ & $79(41.0 \%)$ & 0.61 \\
\hline \multicolumn{5}{|l|}{ Use pesticides indoors } \\
\hline \multicolumn{5}{|l|}{ Do not use pesticides } \\
\hline Yes & $120(25.2 \%)$ & $37(14.0 \%)$ & $82(42.0 \%)$ & - \\
\hline No & $356(74.8 \%)$ & $233(86.0 \%)$ & $111(58.0 \%)$ & $<0.0001$ \\
\hline Pesticides applied by pest control company & - & - & - & - \\
\hline Yes & $233(49.0 \%)$ & $173(64.0 \%)$ & $52(27.0 \%)$ & - \\
\hline No & $243(51.1 \%)$ & $97(36.0 \%)$ & $141(73.0 \%)$ & $<0.0001$ \\
\hline Pesticides applied by director or someone else & - & - & - & - \\
\hline Yes & $104(21.9 \%)$ & $45(17.0 \%)$ & $57(30.0 \%)$ & - \\
\hline No & $372(78.2 \%)$ & $225(83.0 \%)$ & $136(70.0 \%)$ & $<0.0001$ \\
\hline \multicolumn{5}{|l|}{ Use pesticides outdoors } \\
\hline Do not use pesticides & - & - & - & - \\
\hline Yes & $121(25.4 \%)$ & $53(20.0 \%)$ & $66(34.0 \%)$ & - \\
\hline No & $355(74.6 \%)$ & $217(80.0 \%)$ & $127(66.0 \%)$ & $<0.0001$ \\
\hline Pesticides applied by pest control company & - & - & - & - \\
\hline Yes & $206(43.3 \%)$ & $137(51.0 \%)$ & $62(32.0 \%)$ & - \\
\hline No & $270(56.7 \%)$ & $133(49.0 \%)$ & $131(68.0 \%)$ & $<0.0001$ \\
\hline Pesticides applied by director or someone else & - & - & - & - \\
\hline Yes & $149(31.3 \%)$ & $79(29.0 \%)$ & $68(35.0 \%)$ & - \\
\hline No & $327(68.7 \%)$ & $191(71.0 \%)$ & $125(65.0 \%)$ & 0.17 \\
\hline \multicolumn{5}{|l|}{ Frequency of pesticide used indoors } \\
\hline $\begin{array}{l}\text { Pesticides were used, but not sprayed, scattered, or } \\
\text { "bombed" }\end{array}$ & - & - & - & - \\
\hline Yes & $168(35.3 \%)$ & $61(23.0 \%)$ & $104(54.0 \%)$ & \\
\hline No & $308(64.7 \%)$ & $209(77.0 \%)$ & $89(46.0 \%)$ & $<0.0001$ \\
\hline Weekly or monthly & - & - & - & - \\
\hline Yes & $112(23.5 \%)$ & $94(35.0 \%)$ & $12(6.0 \%)$ & - \\
\hline No & $364(76.5 \%)$ & $176(65.0 \%)$ & $181(94.0 \%)$ & $<0.0001$ \\
\hline Yearly or a few times a year & - & - & - & - \\
\hline Yes & $117(24.6 \%)$ & $67(25.0 \%)$ & $50(26.0 \%)$ & - \\
\hline No & $359(75.4 \%)$ & $203(75.0 \%)$ & $143(74.0 \%)$ & 0.79 \\
\hline Whenever pests become problem & - & - & - & - \\
\hline Yes & $49(10.3 \%)$ & $26(10.0 \%)$ & $22(11.0 \%)$ & - \\
\hline No & $427(89.7 \%)$ & $244(90.0 \%)$ & $171(89.0 \%)$ & 0.54 \\
\hline
\end{tabular}


Table 4. Cont.

\begin{tabular}{|c|c|c|c|c|}
\hline Survey Items & $\begin{array}{c}\text { Total } \\
(n=476)\end{array}$ & $\begin{array}{c}\text { Center } \\
(n=270)\end{array}$ & $\begin{array}{c}\text { FCCH } \\
(n=193)\end{array}$ & \\
\hline & $n(\%)$ & $n(\%)$ & $n(\%)$ & $p$-Value ${ }^{1}$ \\
\hline \multicolumn{5}{|l|}{ Frequency of pesticide used outdoors } \\
\hline $\begin{array}{l}\text { Pesticides were used, but not sprayed, scattered, or } \\
\text { "bombed" }\end{array}$ & - & - & - & - \\
\hline Yes & $153(32.1 \%)$ & $74(27.0 \%)$ & $77(40.0 \%)$ & - \\
\hline No & $323(67.9 \%)$ & $196(73.0 \%)$ & $116(60 \%)$ & $<0.0001$ \\
\hline Weekly or monthly & - & - & - & - \\
\hline Yes & $74(15.6 \%)$ & $54(20.0 \%)$ & $16(8.0 \%)$ & - \\
\hline No & $402(84.5 \%)$ & $216(80.0 \%)$ & $177(92.0 \%)$ & $<0.0001$ \\
\hline Yearly or a few times a year & - & - & - & - \\
\hline Yes & $143(30.0 \%)$ & $74(27.0 \%)$ & $67(35.0 \%)$ & - \\
\hline No & $333(70.0 \%)$ & $196(73.0 \%)$ & $126(65.0 \%)$ & 0.09 \\
\hline Whenever pests become a problem & - & - & - & - \\
\hline Yes & $81(17.0 \%)$ & $53(20.0 \%)$ & $27(14.0 \%)$ & - \\
\hline No & $395(83.0 \%)$ & $217(80.0 \%)$ & $166(86.0 \%)$ & 0.11 \\
\hline \multicolumn{5}{|l|}{ Written policy for pesticide use ${ }^{2}$} \\
\hline Yes & $87(21.8 \%)$ & $73(31.0 \%)$ & $12(8.0 \%)$ & - \\
\hline No & $313(78.3 \%)$ & $164(69.0 \%)$ & $138(92.0 \%)$ & $<0.0001$ \\
\hline \multicolumn{5}{|l|}{ Staff are notified before pesticides are applied ${ }^{3}$} \\
\hline Yes & $274(71.7 \%)$ & $166(72.0 \%)$ & $103(75.0 \%)$ & - \\
\hline No & $108(28.3 \%)$ & $66(28.0 \%)$ & $35(25.0 \%)$ & 0.52 \\
\hline \multicolumn{5}{|l|}{ Parents are notified before pesticides are applied ${ }^{4}$} \\
\hline Yes & $129(36.7 \%)$ & $69(29.0 \%)$ & $59(42.0 \%)$ & - \\
\hline No & $223(63.4 \%)$ & $166(71.0 \%)$ & $80(58.0 \%)$ & 0.01 \\
\hline
\end{tabular}

${ }^{1}$ Chi-square tests to analyze differences between Centers and FCCHs; Bolded numbers are $p$-values $<0.05 ;{ }^{2} n=76$ Not applicable, no pesticides are used (missing; omitted from analysis); $n=94$ Not applicable, no pesticides used (missing; omitted from analysis); ${ }^{3} n=94$ Not applicable, no pesticides used (missing; omitted from analysis); ${ }^{4} n=90$ Not applicable, no pesticides are used (missing; omitted from analysis).

See Table 5 for more information about the ECE programs' pest problems and pest control methods.

Table 5. Reported pest problems, pest control methods, applicators, frequency, and related topics $(n=476)$ Oklahoma 2019-2020.

\begin{tabular}{ccccc}
\hline \multirow{2}{*}{ Survey Items } & \multicolumn{2}{c}{ Indoors } & \multicolumn{2}{c}{ Outdoors } \\
\cline { 2 - 5 } & $\boldsymbol{n}$ & $\mathbf{( \% )}$ & $\boldsymbol{n}$ & $\mathbf{( \% )}$ \\
\hline Type of pest problem (listed in alphabetical order) & & & & \\
\hline None & 203 & $(42.7 \%)$ & 159 & $(33.4 \%)$ \\
Ants & 125 & $(26.3 \%)$ & 131 & $(27.7 \%)$ \\
Aphids & 0 & $(0.0 \%)$ & 2 & $(0.4 \%)$ \\
Bed bugs & 7 & $(1.5 \%)$ & 0 & $(0.0 \%)$ \\
Cockroaches & 61 & $(12.8 \%)$ & 6 & $(1.3 \%)$ \\
Crickets & 5 & $(1.1 \%)$ & 0 & $(0.0 \%)$ \\
Fleas & 9 & $(1.9 \%)$ & 14 & $(2.9 \%)$ \\
Head lice & 55 & $(11.6 \%)$ & 0 & $(0.0 \%)$ \\
Mold & 6 & $(1.3 \%)$ & 0 & $(0.0 \%)$ \\
Mosquitoes & 0 & $(0.0 \%)$ & 17 & $(3.6 \%)$ \\
Rodents & 38 & $(8.0 \%)$ & 18 & $(3.8 \%)$ \\
Scorpions & 0 & $(0.0 \%)$ & 6 & $(1.3 \%)$ \\
Snails/Slugs & 3 & $(0.6 \%)$ & 6 & $(1.3 \%)$ \\
Snakes & 0 & $(0.0 \%)$ & 6 & $(1.3 \%)$ \\
\hline
\end{tabular}


Table 5. Cont.

\begin{tabular}{|c|c|c|c|c|}
\hline \multirow{2}{*}{ Survey Items } & \multicolumn{2}{|c|}{ Indoors } & \multicolumn{2}{|c|}{ Outdoors } \\
\hline & $n$ & $(\%)$ & $n$ & $(\%)$ \\
\hline \multicolumn{5}{|l|}{ Type of pest problems (listed in alphabetical order) } \\
\hline Spiders & 88 & $(18.5 \%)$ & 99 & $(20.8 \%)$ \\
\hline Termites & 4 & $(0.8 \%)$ & 5 & $(1.1 \%)$ \\
\hline Ticks & 0 & $(0.0 \%)$ & 3 & $(0.6 \%)$ \\
\hline Wasps/Yellow Jackets & 5 & $(1.1 \%)$ & 147 & $(30.9 \%)$ \\
\hline Other Pests & 15 & $(3.2 \%)$ & 19 & $(30.9 \%)$ \\
\hline \multicolumn{5}{|l|}{ Pest control methods used } \\
\hline Nothing used & 104 & $(21.9 \%)$ & 109 & $(22.9 \%)$ \\
\hline Other methods & 10 & $(2.1 \%)$ & 8 & $(1.7 \%)$ \\
\hline Chemical Pest Control Methods Used & - & - & - & - \\
\hline Sprayed Pesticides & 220 & $(46.2 \%)$ & 224 & $(47.1 \%)$ \\
\hline Poison pellets or powders & 7 & $(1.5 \%)$ & 17 & $(3.6 \%)$ \\
\hline Moth balls & 4 & $(0.8 \%)$ & 0 & $(0.0 \%)$ \\
\hline Applied weed killer & 0 & $(0.0 \%)$ & 60 & $(12.6 \%)$ \\
\hline Non-chemical Pest Control Methods Used & - & - & - & - \\
\hline Cleaned the area & 151 & $(31.7 \%)$ & 79 & $(16.6 \%)$ \\
\hline Sealed cracks/openings & 76 & $(16.0 \%)$ & 35 & $(7.4 \%)$ \\
\hline Removed food sources & 62 & $(13.0 \%)$ & 16 & $(3.4 \%)$ \\
\hline Mouse or rat traps & 53 & $(11.1 \%)$ & 13 & $(2.7 \%)$ \\
\hline Bait Stations or poison traps & 53 & $(11.1 \%)$ & 27 & $(5.7 \%)$ \\
\hline Fixed leaks & 26 & $(5.5 \%)$ & 11 & $(2.3 \%)$ \\
\hline Sticky fly strips & 22 & $(4.6 \%)$ & 12 & $(2.5 \%)$ \\
\hline Installed screens or other barriers & 14 & $(2.9 \%)$ & 11 & $(2.3 \%)$ \\
\hline Cut grass or weeds & 0 & $(0.0 \%)$ & 147 & $(30.9 \%)$ \\
\hline \multicolumn{5}{|l|}{ Who applies pesticides at facility } \\
\hline No one, nothing used & 120 & $(25.2 \%)$ & 121 & $(25.4 \%)$ \\
\hline Pest control company & 233 & $(49.0 \%)$ & 206 & $(43.3 \%)$ \\
\hline Me (participant her/himself) & 54 & $(11.3 \%)$ & 62 & $(13.0 \%)$ \\
\hline Property owner & 18 & $(3.8 \%)$ & 30 & $(6.3 \%)$ \\
\hline Custodial/janitorial staff & 18 & $(3.8 \%)$ & 37 & $(7.8 \%)$ \\
\hline Facility director & 16 & $(3.4 \%)$ & 12 & $(2.5 \%)$ \\
\hline Another staff member & 7 & $(1.5 \%)$ & 12 & $(2.5 \%)$ \\
\hline Someone else (Other) & 4 & $(0.8 \%)$ & 5 & $(1.1 \%)$ \\
\hline Not Sure & 3 & $(0.6 \%)$ & 4 & $(0.8 \%)$ \\
\hline A family member & 2 & $(0.4 \%)$ & 5 & $(1.1 \%)$ \\
\hline \multicolumn{5}{|l|}{ Frequency of pesticide use } \\
\hline Nothing used & 152 & $(31.9 \%)$ & 141 & $(29.6 \%)$ \\
\hline Once a week & 2 & $(0.4 \%)$ & 4 & $(0.8 \%)$ \\
\hline Once a month & 110 & $(23.1 \%)$ & 71 & $(14.9 \%)$ \\
\hline Once a year & 26 & $(5.5 \%)$ & 43 & $(9.0 \%)$ \\
\hline A few times a year & 91 & $(19.1 \%)$ & 101 & $(21.2 \%)$ \\
\hline Whenever pests become a problem & 49 & $(10.3 \%)$ & 81 & $(17.0 \%)$ \\
\hline Pesticides were used, but not sprayed, scattered, or "bombed" & 17 & $(3.6 \%)$ & 13 & $(2.7 \%)$ \\
\hline
\end{tabular}

\section{Discussion}

Understanding the current environmental health policies and practices of ECEs is a critical first step to developing standardized policies and interventions to protect children's health. Our study provides insight into the children's environmental health-related policies and practices of Oklahoma ECEs and reveals some critical opportunities to improve: (1) asthma training, prevention, and control; (2) chemical cleaner and air freshener use; and (3) pest control methods and notification rules when chemical pesticides get used. Centers and FCCHs commonly reported several areas of concern for environmental exposure and 
asthma control, primarily exposure to potentially hazardous chemicals through routine use of bleach, air fresheners, and pesticides.

Our study revealed an important need for asthma-related training and program policies, and practices. Comparable with a study of 40 San Francisco, California centers [50], about half of directors reported receiving any asthma training, and even fewer reported having policies and guidelines for reducing asthma allergens and irritants. Center directors were more likely than $\mathrm{FCCH}$ directors to (1) receive asthma training and (2) report the presence of policies and guidelines for managing asthma medications. Asthma training for ECEs inclusive of asthma trigger avoidance education, particularly for FCCHs, may help ensure programs' preparedness for the prevention and management of asthma flare-ups in this higher risk age group [23].

Our findings indicate that Oklahoma ECE programs routinely use cleaning products and air fresheners that release potentially harmful chemicals known to trigger asthma symptoms and cause adverse respiratory-related health outcomes [10,66-71]. Similar to findings from another study in Washington D.C. [8], most ECE programs in our study reported using bleach to clean their facilities. Centers were more likely to report daily or more frequent bleach use. Under Oklahoma Administrative Code 340:110-3-304. Cleanliness and Sanitation, ECE programs are required to use a household bleach solution or a sanitizer/disinfectant product with a US Environmental Protection Agency (EPA) registration number [58,59]. While effectively disinfecting and sanitizing is important for ECE health, products such as bleach pose risks to children's respiratory health [66]. Alternative methods for sanitizing and disinfecting, such as fragrance-free, non-chlorine, hydrogen peroxide products that have less respiratory toxicity than do bleach or quaternary ammonias [72], may result in overall healthier environments for both children and providers. Additionally, there are national resources available to assist ECE programs in choosing safer disinfectants without compromising a hygienic environment including the (1) US EPA's List $\mathrm{N}$ to identify EPA-registered products that can be used against emerging viral pathogens, such as the Coronavirus, not listed on the product label [73] and (2) the US Department of Labor-Occupational Safety and Health Administration's Hazard Communication Standards to promote chemical safety at ECEs through the provision of information and training to staff regarding chemical labels, safety data sheets, and protocols outlining how to properly handle and store chemicals [74]. In contrast, we found higher rates of air freshener use than reported by Washington D.C. childcare providers [8] and childcare facilities that participated in the evaluation of the Children's Environmental Health Network's EcoHealthy Child Care Checklist [49]; use in FCCHs was particularly high. Air fresheners release more than 100 different hazardous chemicals, including VOCs (e.g., terpenes, such as limonene, alpha-pinene, and beta-pinene; terpenoids, such as linalool; and alpha-terpineol, such as formaldehyde, benzene, toluene, and xylene) and semi-volatile organic compounds (e.g., phthalates) that can contribute to indoor environmental exposure risk [10,67-69]. A pilot study of 14 childcare facilities in Washington D.C. detected six VOCs (i.e., benzene carbon, tetrachloride, chloroform, ethylbenzene, o-xylene, and toluene) inside most childcare facilities with detection frequencies ranging from $71 \%$ to $100 \%$ [8]. Currently, the Oklahoma Licensing Requirements for Child Care Programs [58,59] do not provide any guidance about air fresheners. Education about the potential hazards posed by ECE cleaning behaviors and safer cost-effective alternatives and standardized policies that limit the use of harmful cleaning products and air fresheners may reduce children's exposures in ECE environments [48]. Technical guidance programs for ECEs that assist them in selecting the least toxic options for sanitizing and disinfecting and properly applying and storing products may help to reduce the potential impacts of such products on all children's health.

Similarly, we found much higher usage of broad pesticide application methods (i.e., spray, scatter, or bomb) than reported from a survey conducted in California with 637 centers that found $47.0 \%$ of centers used sprays or foggers to mitigate pest problems, with $20.0 \%$ of those centers applying pesticides weekly or monthly [64]. Pesticides that are 
uncontained may become airborne and leave invisible residues on surfaces such as toys, shades, and walls that can remain for days, posing potential exposure and health risks to children. The majority of Centers in our study used pest control companies to apply pesticides inside their facilities and appeared to schedule the application of pesticides regularly. This practice is inconsistent with recommended IPM strategies to prevent and manage pest problems through removing entry points and access to water and food, only endorsing pesticide use as a last resort and then through contained methods. Pesticide applications inside ECE facilities where children spend most of their time increase their risk of exposure to hazardous residues that may cause exacerbations of asthma symptoms, pediatric cancers, and neurobehavioral and cognitive deficits [75]. Additionally, we found that Centers were less likely to notify parents before applying pesticides. Compared to FCCHs, Centers are housed in larger facilities in commercially zoned areas with more resources than FCCHs, which may explain the higher utilization of pest control companies. Our findings suggest pest control companies do not utilize IPM strategies in ECE environments and instead practice regularly scheduled applications of uncontained, chemical methods as part of their contracts with ECE programs. While California has the Healthy Schools Act [76], which seeks to reduce children's exposures to potentially hazardous toxicants from pesticides in school and ECE environments, few other states including Oklahoma have policies or regulations for ECEs that adequately address children's environmental health. To reduce young children's exposures to pesticides in ECE environments, interventions should address IPM strategies and policies to better manage pests with an emphasis on the reduction of the overall cost of pest control methods with less/non-toxic alternatives. Importantly, adoption of effective pesticide use notification laws to protect the health and safety of employees and families of children attending the ECE.

Study strengths include participation from ECEs across the state and the involvement of both Centers and FCCHs, which enabled us to assess and compare policies and practices between these important, yet distinct, program types. The inclusion of FCCHs adds to the literature, as most research on ECE program environmental health-related policies and practices was conducted with Centers $[50,51,77,78]$. Few studies have examined these policies and practices among FCCHs [52]. To the best of our knowledge, none have assessed differences between Centers and FCCHs.

Study limitations to note include the use of self-reported data that precluded validation of the ECE program directors' responses, which may be subject to social desirability and recall bias. Our study did not objectively measure indoor environmental exposures to the reported chemicals used by ECE programs such as potential exposures to ultrafine and fine particles directly emitted or formed secondarily due to indoor air chemistry. Thus, we cannot determine whether the exposures exceed US EPA and the World Health Organization's indoor air quality guidelines [79]. Although our sample size was moderate, the overall response rate was low, especially for FCCHs, which make up the bulk of the state's licensed ECE programs. It is possible that ECE programs that chose not to participate in this study may be systematically different from those that did and therefore may not represent the overall ECE or FCCH population in Oklahoma. Finally, since we did not include some tribally-owned and operated ECE programs, our study does not adequately assess policies and programs in ECEs owned and operated by sovereign tribal nations in the state. Future researchers may wish to include (1) observations of ECEs and program practices; (2) in-depth data collection about ECE programs' written policies, practices, products, and chemicals used; and (3) measurements of air quality and chemical exposures.

Outside of California [76], few states have policy requirements for ECEs designed to reduce children's exposure to chemicals and other environmental toxicants or to address exposures to environmental toxicants that may originate from or near the ECE facility [80]. Some professional accrediting organizations, such as the NAEYC, have more standards aimed at protecting children's environmental health. NAEYC requires accredited ECE programs to (1) test for radon, (2) use IPM strategies for pest control, and (3) maintain an allergen-free facility [54]. Since only a small (12.2\%) portion of participating ECE programs 
in our study reported NAEYC accreditation, we were unable to assess whether NAEYCaccredited programs' environmental policies and practices differed from those of programs that were not accredited due to the small sample size.

\section{Conclusions}

This study is the first of its kind in Oklahoma and is among very few conducted outside of California and Washington D.C. that have assessed ECE programs' environmental health-related policies and practices. It is the first to evaluate differences in these policies and practices between Centers and FCCHs. The findings highlight the need for interventions that will improve rates of asthma training, reduce the presence of asthma triggers, and promote the use of less/non-toxic pest control methods such as IPM. Given the many differences we found in policies and practices between Centers and FCCHs, it is critical that interventions be tailored to these contexts and address the most salient drivers of organizational and/or provider behaviors in these settings. Interdisciplinary collaboration among clinicians, public health professionals, education and child development specialists, and ECE providers themselves may enhance future research to further understand the drivers and impacts of environmental exposures in ECE programs and develop interventions to promote healthier ECE environments in both Centers and FCCHs.

Supplementary Materials: The following are available online at https://www.mdpi.com/article/10 .3390 /ijerph18168491/s1, Figure S1: Communities and Classroom Health Survey.

Author Contributions: Conceptualization, C.D.Q., B.W., S.B.S. and A.L.S.; Data curation, C.D.Q. and B.W.; Formal analysis, C.D.Q. and A.L.S.; Funding acquisition, B.W. and S.B.S.; Methodology, C.D.Q., B.W., S.B.S. and A.L.S.; Supervision, A.L.S.; Writing-original draft, C.D.Q. and A.L.S.; Writingreview and editing, B.W., M.S.W., S.B.S., J.C., M.G., and L.S. All authors have read and agreed to the published version of the manuscript.

Funding: This research was supported by the Administration for Children and Families (ACF) of the United States (U.S.) Department of Health and Human Services (HHS) as part of a financial assistance award (Grant \#: 90YR0115) totaling \$25,000 with 100 percent funded by ACF/HHS. The contents are those of the author(s) and do not necessarily represent the official views of, nor an endorsement, by ACF/HHS, or the U.S. Government. For more information, please visit the ACF website, Administrative and National Policy Requirements. Funding was also provided by the University of Oklahoma Health Sciences Center (OUHSC) College of Allied Health Student Research and Creativity Grant and the Department of Nutritional Sciences. Additional support for Cassandra D. Querdibitty was provided by the Hudson Fellowship Program awarded to the University of Oklahoma Hudson College of Public Health. The content is solely the responsibility of the authors and does not necessarily represent the official views of the Hudson College of Public Health or the Hudson Fellowship Program. Additional support for this study was provided by the Oklahoma Shared Clinical and Translational Resources (U54GM104938) with an Institutional Development Award (IDeA) from NIGMS. The content is solely the responsibility of the authors and does not necessarily represent the official views of the National Institutes of Health.

Institutional Review Board Statement: The study was reviewed and deemed to be exempt by the Institutional Review Board of the University of Oklahoma Health Sciences Center because only data about ECE programs (i.e., no personal data) were being reported.

Informed Consent Statement: Informed consent was waived due to data being program-level, not individual-level; thus, this study was not considered as human-subjects research.

Data Availability Statement: The data presented in this study are available on request from the corresponding author. The data are not publicly available.

Acknowledgments: We are grateful to our community partners at the Oklahoma Department of Human Services, the Office of Head Start Collaboration, the Oklahoma State Department of Health, the Oklahoma Association for the Education of Young Children, and the Oklahoma Child Care Association. We also thank Nancy Rader Inhofe, Daisy Butzer, and the members of our study advisory council (Kay Floyd, Bryce Lowery, Dipti Dev, Diane Horm, Denise Finneran, and Jennifer Graef-Downard). 
Conflicts of Interest: The authors declare no conflict of interest and the funders had no role in the design of the study; in the collection, analyses, or interpretation of data; in the writing of the manuscript, or in the decision to publish the results.

\section{References}

1. Laughlin, L. Who's Minding the Kids? Child Care Arrangements: Spring 2011; U.S. Census Bureau: Washington, DC, USA, 2013; pp. 70-135.

2. Committee on Early Childhood, Adoption, and Dependent Care. Quality Early Education and Child Care From Birth to Kindergarten. Pediatrics 2017, 140, 187-191. [CrossRef]

3. Hoang, T.; Castorina, R.; Gaspar, F.; Maddalena, R.; Jenkins, P.L.; Zhang, Q.; McKone, T.E.; Benfenati, E.; Shi, A.Y.; Bradman, A. VOC exposures in California early childhood education environments. Indoor Air 2017, 27, 609-621. [CrossRef]

4. Bradman, A.; Gaspar, F.; Castorina, R.; Tong-Lin, E.; Mckone, T. Environmental Exposures in Early Childhood Education Environments. Available online: https://ww2.arb.ca.gov/sites/default/files/classic//research/apr/past/08-305.pdf (accessed on 15 November 2020).

5. Bradman, A.; Castorina, R.; Gaspar, F.; Nishioka, M.; Colon, M.; Weathers, W.; Egeghy, P.P.; Maddalena, R.; Williams, J.; Jenkins, P.L.; et al. Flame retardant exposures in California early childhood education environments. Chemosphere 2014, 116, 61-66. [CrossRef]

6. Breysse, P.; Farr, N.; Galke, W.; Lanphear, B.; Morley, R.; Bergofsky, L. The relationship between housing and health: Children at risk. Environ. Health Perspect. 2004, 112, 1583-1588. [CrossRef]

7. Gaspar, F.W.; Castorina, R.; Maddalena, R.L.; Nishioka, M.G.; McKone, T.E.; Bradman, A. Phthalate exposure and risk assessment in California child care facilities. Environ. Sci. Technol. 2014, 48, 7593-7601. [CrossRef]

8. Quiros-Alcala, L.; Wilson, S.; Witherspoon, N.; Murray, R.; Perodin, J.; Trousdale, K.; Raspanti, G.; Sapkota, A. Volatile organic compounds and particulate matter in child care facilities in the District of Columbia: Results from a pilot study. Environ. Res. 2016, 146, 116-124. [CrossRef] [PubMed]

9. Tulve, N.S.; Jones, P.A.; Nishioka, M.G.; Fortmann, R.C.; Croghan, C.W.; Zhou, J.Y.; Fraser, A.; Cavel, C.; Friedman, W. Pesticide measurements from the first national environmental health survey of child care centers using a multi-residue GC/MS analysis method. Environ. Sci. Technol. 2006, 40, 6269-6274. [CrossRef]

10. Nazaroff, W.W.; Weschler, C.J. Cleaning products and air fresheners: Exposure to primary and secondary air pollutants. Atmos. Environ. 2004, 38, 2841-2856. [CrossRef]

11. Neal, R.; Spurlock, F.; Segawa, R. Annual Report on Volatile Organic Compound Emissions from Pesticides: Emissions for 1990-2010. Available online: https://www.cdpr.ca.gov/docs/emon/vocs/vocproj/2017_voc_annual_report.pdf (accessed on 1 August 2021).

12. Anderson, J.O.; Thundiyil, J.G.; Stolbach, A. Clearing the air: A review of the effects of particulate matter air pollution on human health. J. Med. Toxicol. 2012, 8, 166-175. [CrossRef]

13. Kim, K.-H.; Kabir, E.; Kabir, S. A review on the human health impact of airborne particulate matter. Environ. Int. 2015, 74, 136-143. [CrossRef] [PubMed]

14. Burtscher, H.; Schuepp, K. The occurrence of ultrafine particles in the specific environment of children. Paediatr. Respir. Rev. 2012, 13, 89-94. [CrossRef]

15. Salvi, S. Health effects of ambient air pollution in children. Paediatr. Respir. Rev. 2007, 8, 275-280. [CrossRef] [PubMed]

16. ATDSR. Toxicological Profile for Carbon Tetrachloride. Available online: https://www.atsdr.cdc.gov/toxprofiles/tp30.pdf (accessed on 12 January 2021).

17. United States Environmental Protection Agency. Exposure Factors Handbook 2011 Edition (Final). Available online: https: / / www.nrc.gov / docs / ML1400/ML14007A666.pdf (accessed on 20 January 2021).

18. Goldizen, F.C.; Sly, P.D.; Knibbs, L.D. Respiratory effects of air pollution on children. Pediatr. Pulmonol. 2016, 51, 94-108. [CrossRef] [PubMed]

19. Harving, H.; Dahl, R.; Mølhave, L. Lung Function and Bronchial Reactivity in Asthmatics during Exposure to Volatile Organic Compounds. Am. Rev. Respir. Dis. 1991, 143, 751-754. [CrossRef] [PubMed]

20. Koren, H.S.; Graham, D.E.; Devlin, R.B. Exposure of Humans to a Volatile Organic Mixture. III. Inflammatory Response. Arch. Environ. Health Int. J. 1992, 47, 39-44. [CrossRef] [PubMed]

21. Mortimer, K.; Neugebauer, R.; Lurmann, F.; Alcorn, S.; Balmes, J.; Tager, I. Air pollution and pulmonary function in asthmatic children: Effects of prenatal and lifetime exposures. Epidemiology 2008, 19, 550-557. [CrossRef]

22. Norbäck, D.; Björnsson, E.; Janson, C.; Widström, J.; Boman, G. Asthmatic symptoms and volatile organic compounds, formaldehyde, and carbon dioxide in dwellings. Occup. Environ. Med. 1995, 52, 388-395. [CrossRef] [PubMed]

23. Hollenbach, J.P.; Cloutier, M.M. Childhood Asthma Management and Environmental Triggers. Pediatr. Clin. N. Am. 2015, 62, 1199-1214. [CrossRef]

24. Jia, C.; Batterman, S.; Godwin, C. VOCs in industrial, urban and suburban neighborhoods—Part 2: Factors affecting indoor and outdoor concentrations. Atmos. Environ. 2008, 42, 2101-2116. [CrossRef] 
25. Wieslander, G.; Norbäck, D.; Björnsson, E.; Janson, C.; Boman, G. Asthma and the indoor environment: The significance of emission of formaldehyde and volatile organic compounds from newly painted indoor surfaces. Int. Arch. Occup. Environ. Health 1997, 69, 115-124. [CrossRef]

26. Cox, S.S.; Little, J.C.; Hodgson, A.T. Predicting the Emission Rate of Volatile Organic Compounds from Vinyl Flooring. Environ. Sci. Technol. 2002, 36, 709-714. [CrossRef]

27. Wilke, O.; Jann, O.; Brödner, D. VOC- and SVOC-emissions from adhesives, floor coverings and complete floor structures. Indoor Air 2004, 14 (Suppl. 8), 98-107. [CrossRef]

28. Jo, W.-J.; Sohn, J.-Y. The effect of environmental and structural factors on indoor air quality of apartments in Korea. Build. Environ. 2009, 44, 1794-1802. [CrossRef]

29. Singer, B.C.; Destaillats, H.; Hodgson, A.T.; Nazaroff, W.W. Cleaning products and air fresheners: Emissions and resulting concentrations of glycol ethers and terpenoids. Indoor Air 2006, 16, 179-191. [CrossRef] [PubMed]

30. Parks, J.; McCandless, L.; Dharma, C.; Brook, J.; Turvey, S.E.; Mandhane, P.; Becker, A.B.; Kozyrskyj, A.L.; Azad, M.B.; Moraes, T.J. Association of use of cleaning products with respiratory health in a Canadian birth cohort. CMAJ 2020, 192, E154-E161. [CrossRef] [PubMed]

31. Gordon, S.M.; Callahan, P.J.; Nishioka, M.G.; Brinkman, M.C.; O’Rourke, M.K.; Lebowitz, M.D.; Moschandreas, D.J. Residential environmental measurements in the National Human Exposure Assessment Survey (NHEXAS) pilot study in Arizona: Preliminary results for pesticides and VOCs. J. Expo. Sci. Environ. Epidemiol. 1999, 9, 456-470. [CrossRef]

32. Ott, W.R.; Roberts, J.W. Everyday exposure to toxic pollutants. Sci. Am. 1998, 278, 86-91. [CrossRef]

33. Chin, J.Y.; Godwin, C.; Parker, E.; Robins, T.; Lewis, T.; Harbin, P.; Batterman, S. Levels and sources of volatile organic compounds in homes of children with asthma. Indoor Air 2014, 24, 403-415. [CrossRef]

34. Beamer, P.; Castano, A.; Leckie, J. Vertical Profile Particulate Matter Measurements in a California Daycare. Available online: https:/ / www.irbnet.de/daten/iconda/CIB6560.pdf (accessed on 30 July 2021).

35. Gruchalla, R.S.; Pongracic, J.; Plaut, M.; Evans, R., III; Visness, C.M.; Walter, M.; Crain, E.F.; Kattan, M.; Morgan, W.J.; Steinbach, S.; et al. Inner City Asthma Study: Relationships among sensitivity, allergen exposure, and asthma morbidity. J. Allergy Clin. Immunol. 2005, 115, 478-485. [CrossRef] [PubMed]

36. Torjusen, E.N.; Diette, G.B.; Breysse, P.N.; Curtin-Brosnan, J.; Aloe, C.; Matsui, E.C. Dose-response relationships between mouse allergen exposure and asthma morbidity among urban children and adolescents. Indoor Air 2013, 23, 268-274. [CrossRef]

37. Sheehan, W.J.; Rangsithienchai, P.A.; Wood, R.A.; Rivard, D.; Chinratanapisit, S.; Perzanowski, M.S.; Chew, G.L.; Seltzer, J.M.; Matsui, E.C.; Phipatanakul, W. Pest and allergen exposure and abatement in inner-city asthma: A work group report of the American Academy of Allergy, Asthma \& Immunology Indoor Allergy / Air Pollution Committee. J. Allergy Clin. Immunol. 2010, 125, 575-581. [CrossRef]

38. Morgan, W.J.; Crain, E.F.; Gruchalla, R.S.; O'Connor, G.T.; Kattan, M.; Evans, R.; Stout, J.; Malindzak, G.; Smartt, E.; Plaut, M.; et al. Results of a Home-Based Environmental Intervention among Urban Children with Asthma. N. Eng. J. Med. 2004, 351, 1068-1080 [CrossRef]

39. Arbes, S.J.; Sever, M.; Mehta, J.; Collette, N.; Thomas, B.; Zeldin, D.C. Exposure to indoor allergens in day-care facilities: Results from 2 North Carolina counties. J. Allergy Clin. Immunol. 2005, 116, 133-139. [CrossRef] [PubMed]

40. Perry, T.T.; Vargas, P.A.; Bufford, J.; Feild, C.; Flick, M.; Simpson, P.M.; Hamilton, R.G.; Jones, S.M. Classroom aeroallergen exposure in Arkansas head start centers. Ann. Allergy Asthma Immunol. 2008, 100, 358-363. [CrossRef]

41. Phipatanakul, W.; Cronin, B.; Wood, R.A.; Eggleston, P.A.; Shih, M.C.; Song, L.; Tachdjian, R.; Oettgen, H.C. Effect of environmental intervention on mouse allergen levels in homes of inner-city Boston children with asthma. Ann. Allergy Asthma Immunol. 2004, 92, 420-425. [CrossRef]

42. Chaudhuri, N. Interventions to improve children's health by improving the housing environment. Rev. Environ. Health 2004, 19, 197-222. [CrossRef] [PubMed]

43. Katial, R.K. Cockroach allergy. Immunol. Allergy Clin. N. Am. 2003, 23, 483-499. [CrossRef]

44. Eggleston, P.A. Methods and effectiveness of indoor environmental control. Ann. Allergy Asthma Immunol. 2001, 87, 44-47. [CrossRef]

45. Fournier, A.; Johnson, T. Implementation of Pilot Integrated pest Management Programs in Indiana Schools and Child Care Facilities, Executive Summary; IPM School Technical Resource Center: West Lafayette, IN, USA, 2003.

46. Piper, C.; Owens, K. Are schools making the grade? School districts nationwide adopt safer pest management policies. Pestic. You 2002, 22, 11-20.

47. Anderson, M.; Glynn, T.; Enache, A. EPA Region 2 Pesticides in Child Care Initiative 2010 Staten Island Pilot Project Final Report. Available online: https:/ / www.epa.gov/sites/production/files/documents/StatenPilot2010.pdf (accessed on 12 November 2020).

48. Amoah, A.O.; Witherspoon, N.O.; Pérodin, J.; Paulson, J.A. Findings from a pilot environmental health intervention at early childhood centers in the District of Columbia. J. Public Health 2016, 38, e209-e217. [CrossRef]

49. Gilden, R.; McElroy, K.; Friedmann, E.; Witherspoon, N.O.; Paul, H. Evaluation of the children's environmental health network's environmental stewardship checklist responses. J. Environ. Health 2015, 77, 22-29. [PubMed] 
50. Young, C.A.; Stookey, J.; Patel, A.I.; Chan, C.; Evans, J.; Cohn, K.; Agana, L.; Yen, I.H.; Fernandez, A.; Cabana, M.D. San Francisco childcare centers' preparedness in the prevention and management of asthma among preschool-aged children. J. Asthma 2016, 53, 691-698. [CrossRef] [PubMed]

51. Alkon, A.; Nouredini, S.; Swartz, A.; Sutherland, A.M.; Stephens, M.; Davidson, N.A.; Rose, R. Integrated Pest Management Intervention in Child Care Centers Improves Knowledge, Pest Control, and Practices. J. Pediatr. Health Care 2016, 30 , e27-e41. [CrossRef] [PubMed]

52. Stephens, M.; Hazard, K.; Moser, D.; Cox, D.; Rose, R.; Alkon, A. An Integrated Pest Management Intervention Improves Knowledge, Pest Control, and Practices in Family Child Care Homes. Int. J. Environ. Res. Public Health 2017, 14, 1299. [CrossRef]

53. Goveia, M.; Shaikh, N.; Windham, G.; Bembom, O.; Feldman, K.; Kreutzer, R. Asthma-Related Environmental Practices and Asthma Awareness in California Child Care Centers. Pediatr. Asthma Allergy Immunol. 2005, 18, 12-24. [CrossRef]

54. Attar, K.; Paul, H. Let's Clear the Air: Creating Eco-Friendly, Healthy Learning Environments. Available online: https://www. naeyc.org/resources/pubs/tyc/oct2019/eco-friendly-environment (accessed on 2 February 2020).

55. University of California Agriculture and Natural Resources. What Is Integrated Pest Management (IPM)? Available online: https:/ / www2.ipm.ucanr.edu/What-is-IPM/ (accessed on 5 April 2021).

56. Center for Disease Control and Prevention. Table C1 Child Current Asthma Prevalence and Weighted Number by State or Territory: Behavioral Risk Factor Surveillance System 2016. Available online: https://www.cdc.gov/asthma/brfss/2016/child/C1_508.pdf (accessed on 10 March 2021).

57. Centers for Disease Control and Prevention. AsthmaStats Uncontrolled Asthma among Children, 2012-2014. Available online: https:/ / www.cdc.gov/asthma/asthma_stats / documents / AsthmaStats_Asthma_Uncontrolled_Child_PDF_Cleared_H.pdf (accessed on 15 May 2021).

58. Oklahoma Department of Human Services. Licensing Requirements for Child Care Programs. Available online: http://www. okdhs.org/OKDHS\%20Publication\%20Library/14-05.pdf (accessed on 25 March 2020).

59. Oklahoma Department of Human Services. Licensing Requirements for Family Child Care Homes and Large Child Care Homes. Available online: http:/ / www.okdhs.org/OKDHS\%20Publication\%20Library/86-104.pdf (accessed on 20 November 2020).

60. Williams, B.D.; Sisson, S.B.; Dev, D.A.; Lowery, B.; Horm, D.; Campbell, J.; Finneran, D.; Graef-Downard, J.; Whaley, L. Associations between Community Built Environments with Early Care and Education Classroom Physical Activity Practices and Barriers. Int. J. Environ. Res. Public Health 2021, 18, 6524. [CrossRef] [PubMed]

61. Harris, P.A.; Taylor, R.; Thielke, R.; Payne, J.; Gonzalez, N.; Conde, J.G. Research electronic data capture (REDCap)—A metadatadriven methodology and workflow process for providing translational research informatics support. J. Biomed. Inform. 2009, 42, 377-381. [CrossRef]

62. Harris, P.A.; Taylor, R.; Minor, B.L.; Elliott, V.; Fernandez, M.; O’Neal, L.; McLeod, L.; Delacqua, G.; Delacqua, F.; Kirby, J.; et al. The REDCap consortium: Building an international community of software platform partners. J. Biomed. Inform. $2019,95,103208$. [CrossRef]

63. Young, C.A.; Chan, C.; Stookey, J.; Patel, A.I.; Evans, J.; Cohn, K.; Agana, L.; Yen, I.H.; Fernandez, A.; Cabana, M.D. Development of a Tool to Evaluate Asthma Preparedness and Management in Child-Care Centers. Pediatr. Allergy Immunol. Pulmonol. 2015, 28, 121-128. [CrossRef]

64. The Center for Children's Environmental Health Research. Pest Management and Pesticide Use in California Child Care Centers. Available online: https:/ / cerch.berkeley.edu/sites/default/files / pest_mangement_and_pesticide_use_in_california_child_care_ centers.pdf (accessed on 30 July 2019).

65. Holm, S. A simple sequentially rejective multiple test procedure. Scand. J. Stat. 1979, 6, 65-70.

66. Agana, L.; Melgoza, C. Bleach Exposure in Child Care Settings: Strategies for Elimination or Reduction. Available online: https: //www.asthmacommunitynetwork.org/sites/default/files/resource_files/ATF-Final-Project-Report_March-2011.pdf (accessed on 27 March 2020).

67. Uhde, E.; Schulz, N. Impact of room fragrance products on indoor air quality. Atmos. Environ. 2015, 106, 492-502. [CrossRef]

68. Kim, S.; Hong, S.H.; Bong, C.K.; Cho, M.H. Characterization of air freshener emission: The potential health effects. J. Toxicol. Sci. 2015, 40, 535-550. [CrossRef]

69. Steinemann, A. Volatile emissions from common consumer products. Air Qual. Atmos. Health 2015, 8, 273-281. [CrossRef]

70. Trantallidi, M.; Dimitroulopoulou, C.; Wolkoff, P.; Kephalopoulos, S.; Carrer, P. EPHECT III: Health risk assessment of exposure to household consumer products. Sci. Total Environ. 2015, 536, 903-913. [CrossRef] [PubMed]

71. Nørgaard, A.W.; Kudal, J.D.; Kofoed-Sørensen, V.; Koponen, I.K.; Wolkoff, P. Ozone-initiated VOC and particle emissions from a cleaning agent and an air freshener: Risk assessment of acute airway effects. Environ. Int. 2014, 68, 209-218. [CrossRef] [PubMed]

72. Holm, S.M.; Leonard, V.; Durrani, T.; Miller, M.D. Do we know how best to disinfect child care sites in the United States? A review of available disinfectant efficacy data and health risks of the major disinfectant classes. Am. J. Infect. Control 2019, 47, 82-91. [CrossRef]

73. United States Environmental Protection Agency. About List N: Disinfectants for Coronavirus (COVID-19). Available online: https:/ / www.epa.gov / coronavirus / about-list-n-disinfectants-coronavirus-covid-19-0 (accessed on 27 July 2021).

74. United States Department of Labor. Hazard Communication. Available online: https://www.osha.gov/hazcom (accessed on 27 July 2021).

75. Roberts, J.R.; Karr, C.J. Pesticide exposure in children. Pediatrics 2012, 130, e1765-e1788. [CrossRef] 
76. Geiger, C.; Tootelian, D. Healthy Schools Act spurs integrated pest management in California public schools. Calif. Agric. 2005, 59, 235-241. [CrossRef]

77. Mir, D.F.; Finkelstein, Y.; Tulipano, G.D. Impact of integrated pest management (IPM) training on reducing pesticide exposure in Illinois childcare centers. NeuroToxicol. 2010, 31, 621-626. [CrossRef]

78. Alkon, A.; Kalmar, E.; Leonard, V.; Flint, M.L.; Kuo, D.; Davidson, N.; Bradman, A. Development and Evaluation of an Integrated Pest Management Toolkit for Child Care Providers. Early Child. Res. Pract. 2012, 14, n2.

79. Al-Hemoud, A.; Al-Awadi, L.; Al-Khayat, A.; Behbehani, W. Streamlining IAQ guidelines and investigating the effect of door opening/closing on concentrations of VOCs, formaldehyde, and NO2 in office buildings. Build. Environ. 2018, 137, 127-137. [CrossRef]

80. Hudson, G.; Miller, G.G.; Seikel, K. Regulations, policies, and guidelines addressing environmental exposures in early learning environments: A review. J. Environ. Health 2014, 76, 24-34. [PubMed] 